J. Phys. B: At. Mol. Opt. Phys., 37, (2004), p.2585 - 2592.

ISSN: 0953-4075(04)75545-5 ISSN 1361-6455 (Online)

doi:10.1088/0953-4075/37/12/013

(C) IOP Publishing Ltd

http://journals.iop.org/

http://www.iop.org/EJ/journal/JPhysB

This is an un-copyedited version of an article accepted for publication in J. Phys. B: At. Mol. Opt. Phys. IOP

Publishing Ltd is not responsible for any errors or omissions in this version of the manuscript or any version derived from it. The definitive publisher authenticated version is available online at doi:10.1088/0953-4075/37/12/013.

\title{
Resolution and accuracy of resonances in $R$-matrix cross sections
}

\author{
Franck Delahaye ${ }^{1}$, Sultana N Nahar ${ }^{1}$, Anil K Pradhan ${ }^{1}$ and Hong Lin Zhang ${ }^{2}$ \\ ${ }^{1}$ Department of Astronomy, Ohio State University, Columbus, OH 43210, USA \\ ${ }^{2}$ Applied Physics Division, Los Alamos National Laboratory, Los Alamos, NM 87545, USA
}

\begin{abstract}
We investigate the effect of resonances in photoionization and recombination cross sections computed using the $R$-matrix method. Photoionization and recombination rates derived from high-resolution cross sections for oxygen ions are compared with earlier works with less resolution and accuracy, such as in the widely used Opacity Project data. We find significant differences in photoionization rates for O II metastable states, averaged over Planck functions corresponding to ionizing radiation fields, with respect to the intrinsic accuracy of the calculations and improved resolution. Furthermore, for highly charged ions other physical effects are also important. Recombination rate coefficients, averaged over a Maxwellian distribution, are extremely sensitive to the position and resolution of near-threshold resonances and radiation damping, in (e $+\mathrm{O}$ VII $) \leftrightarrow \mathrm{O} V \mathrm{I}+h v$. Surprisingly, however, the effect on the monochromatic and the mean Rosseland and Planck bound-free opacities is relatively small, but may be potentially significant.
\end{abstract}

\section{Introduction}

A precise treatment of resonances has always been of prime concern in coupled channel calculations for electron impact excitation, photoionization and recombination. Sophisticated methods have been employed to resolve and/or fit resonances in order that cross sections and averaged rates, the quantities of interest in applications, may be computed accurately. One of the main reasons that the powerful $R$-matrix method (Burke et al 1971, Hummer et al 1993, Burke and Berrington 1993) has been very successful is because it enables efficient calculations at a large number of energies to affect such a resolution. Following a single diagonalization of the $R$-matrix for each total electron-ion symmetry $L S \pi$ or $J \pi$, cross sections may be obtained at any number of energies. The $R$-matrix method and its relativistic variant the Breit-Pauli $R$-matrix method (BPRM, Berrington et al 1995), have been employed in large scale calculations in the Opacity Project (OP) (Seaton et al 1994), The Opacity Project Team 1995/1996) and the Iron Project (IP) (Hummer et al 1993) for radiative and collisional cross sections for most astrophysically abundant elements, nearly 200 atoms and ions. The OP/IP work involves photoionization and electron scattering cross sections, as well as transition probabilities. The $R$-matrix method (and BPRM) has been further extended to a unified treatment of electron-ion recombination including radiative and di-electronic recombination (RR and DR) in an ab initio manner, which is also self-consistent with 
$R$-matrix photoionization cross sections (Nahar and Pradhan 1992 1994a, Zhang and Pradhan 1997, Zhang et al 1999, Nahar and Pradhan 2003a). A crucial aspect of the unified method for (e + ion) recombination is the high-resolution imperative for the derivation of accurate rate coefficients. However, some physical effects are also very important in determining the accuracy of computed parameters as we demonstrate in this paper. The present work also addresses some of the issues and results described by Ramirez and Bautista (2002).

\section{Results}

The effect of resolution and accuracy of resonances depends on physical processes. The height of photoionization resonances is theoretically unbounded, unlike electron-ion excitation cross sections where it is limited by the unitarity constraint on the scattering matrix. Therefore, resonances in photoionization cross sections may approach unphysical heights if all relevant physical effects are not carefully considered. A complete resolution of resonances in photoionization cross sections, particularly for higher $n$ and $\ell$, is difficult to achieve. Neither can the calculated resonances be individually verified by experiments, since the measured cross sections are averages over the beam width and shape. Nevertheless, $R$-matrix calculations for photoionization cross sections have been experimentally verified, some computed in $L S$ coupling prior to experiments. For example, we note the extremely detailed comparison between $R$-matrix cross sections and recent experiments on synchrotron based light sources reported by the Reno/Berkeley group for O II (Covington et al 2001), the Aarhus group for C II (Kjeldsen et al 1999, Nahar 2002) and the Paris group for O ions (Champeaux et al 2003, Nahar 2004). Near-threshold resonances need to be carefully resolved, often with relativistic fine structure. In the relativistic Fe XVII photorecombination calculations including 359 bound levels with $n<10$, Zhang et al (2001) explicitly delineate 2985 resonances in a very small near-threshold region 0-5 $\mathrm{eV}$.

\subsection{Photoionization cross sections and rates}

In figure 1 we compare high-resolution $R$-matrix photoionization cross sections for the ground and two metastable states of O II, $2 \mathrm{~s}^{2} 2 \mathrm{p}^{3}\left({ }^{4} \mathrm{So},{ }^{2} \mathrm{Do},{ }^{2} \mathrm{Po}\right.$ ), with the OP data from Topbase (Cunto et al 1993, http://vizier.u-strasbg.fr/OP.html, http://heasarc.gsfc.nasa.gov/ topbase/home.html). The OP cross sections were the most accurate attempt at the time of computations to resolve resonances. In general, instead of a constant energy mesh, a quantum-defect mesh was employed to enable similar resolution of resonances along a Rydberg series as they get narrower with increasing $n$. The quantum-defect mesh ensures a pre-determined number of points (usually 100) to resolve each range of effective quantum number $(v, v+1)$. Although this does not guarantee complete resolution and the OP cross sections may still be under-resolved for certain applications such as monochromatic spectral modelling, they are considerably better than those with constant energy mesh. 

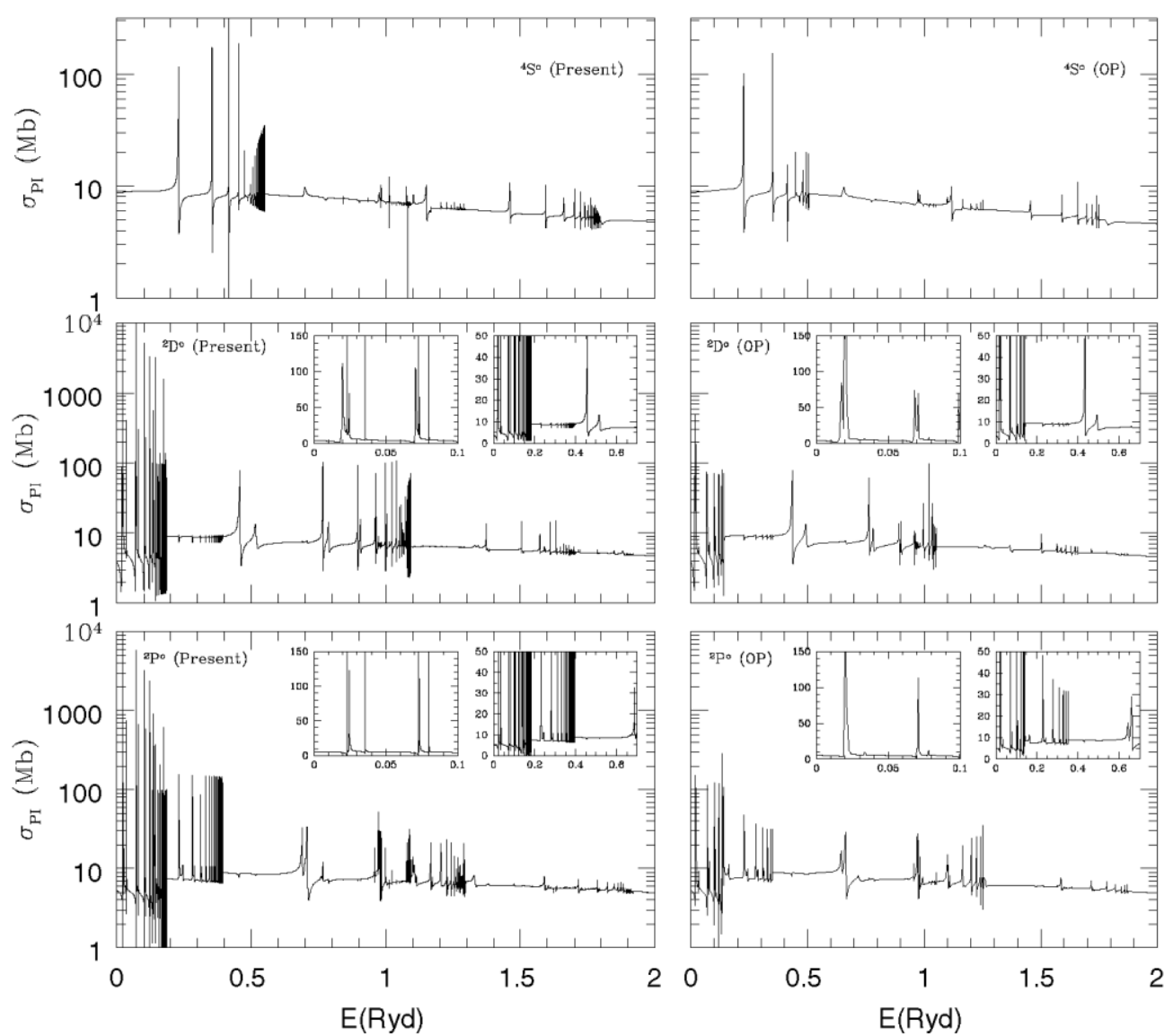

Figure 1. Photoionization cross sections of the ground-state $1 s^{2} 2 s^{2} 2 p^{3}\left({ }^{4} S o\right)$ and two metastable states $1 \mathrm{~s}^{2} 2 \mathrm{~s}^{2} 2 \mathrm{p}^{3}\left({ }^{2} \mathrm{Po},{ }^{2} \mathrm{Do}\right.$ ) of O II (from top to bottom). The present high-resolution data are on the left and the Opacity Project data from Topbase are on the right. The insets show the shift in resonance positions and the effect of resolution in the near-threshold region.

The new results reported in this work are obtained as in the recent calculations for a number of oxygen ions, O II-O V, using a more extensive eigenfunction expansion for the target ion than in the OP work; they are in good detailed agreement with recent experimental measurements (Nahar 2004). Extensive resonance structures are delineated, especially in the near-threshold region. As figure 1 shows, resonances are more completely resolved in the present calculations, including those along a Rydberg series converging on to excited thresholds. Moreover, one important point is that the resonance positions are at somewhat different energies compared to the corresponding OP data.

Photoionization rates $\Gamma_{\mathrm{PI}}$ are computed using both the present and the OP cross sections, assuming ionizing radiation fields from a blackbody source $\left(B_{v}(T)\right)$ at radiation temperatures $T$ represented by a Planck function

$$
\begin{aligned}
& \Gamma_{\mathrm{PI}}=W \int \frac{4 \pi B_{v}(T) \sigma_{\mathrm{PI}}(v)}{h v} \mathrm{~d} v \\
& B_{v}(T)=\left(2 h v^{3} / c^{2}\right)\left[\exp \left(h v / k_{\mathrm{B}} T\right)-1\right]^{-1}
\end{aligned}
$$


where $W$ is a dilution factor (set to 1 for the present study) and $O_{\mathrm{PI}}$ is the photoionization cross section.

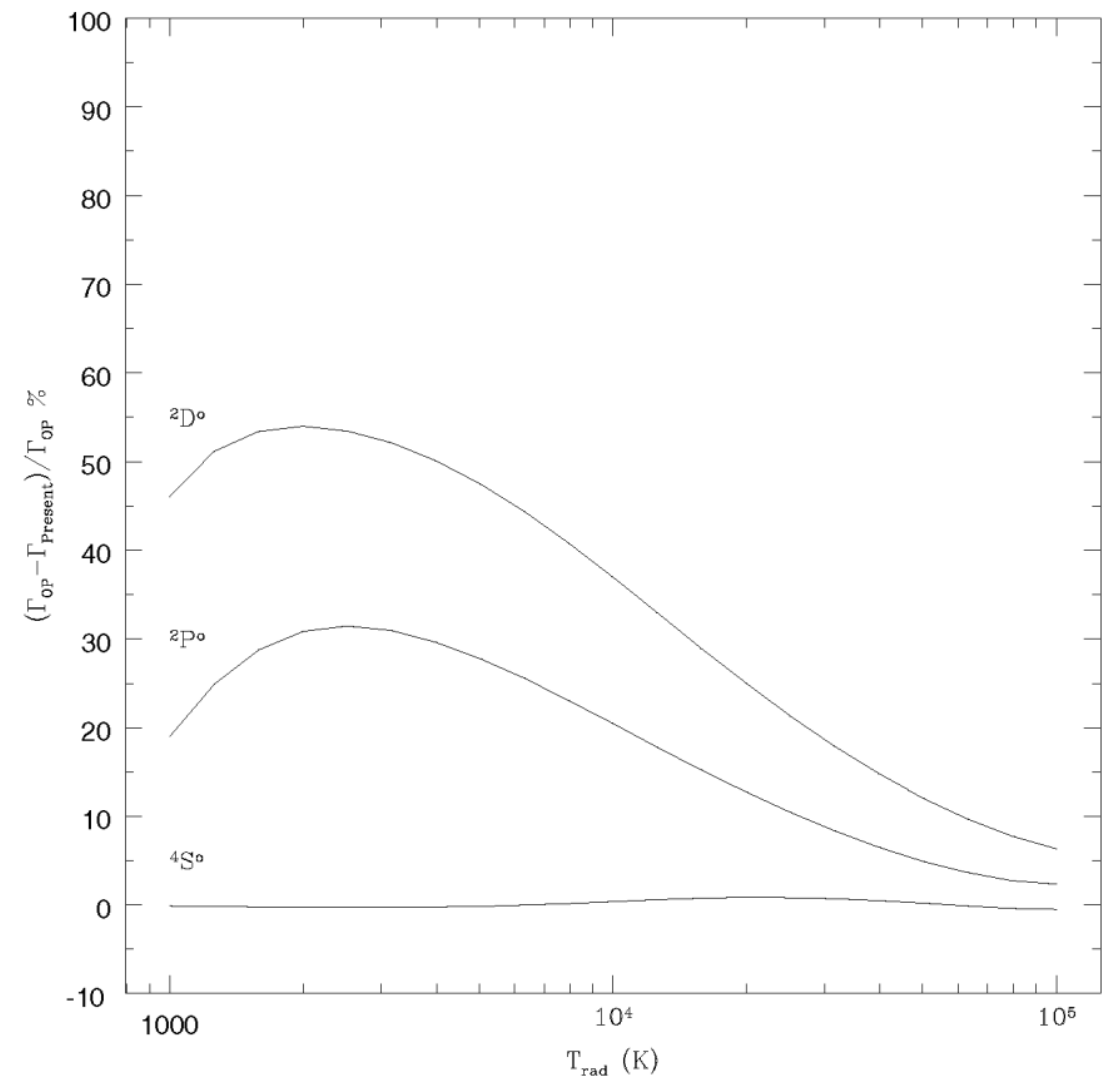

Figure 2. Percentage differences in photoionization rates for the ground state $1 \mathrm{~s}^{2} 2 \mathrm{~s}^{2} 2 \mathrm{p}^{3}\left({ }^{4} \mathrm{~S}^{\mathrm{o}}\right)$ and metastable states $1 \mathrm{~s}^{2} 2 \mathrm{~s}^{2} 2 \mathrm{p}^{3}\left({ }^{2} \mathrm{P}^{\mathrm{o}},{ }^{2} \mathrm{D}^{\circ}\right)$ of O II, at radiation temperatures $\mathrm{T}_{\mathrm{rad}}$.

We can see in figure 1 that the ground-state cross sections agree well. The position of the resonances is identical. Their heights differ but, as shown below, it should not affect the rates significantly because the area under these peaks is not significant since the resonances are narrow. For the two metastable states a shift in the position of the resonances can be seen. The difference in resolution also modifies the computed shape of the resonances, especially for the first ones. These two factors, resolution and accuracy of the position of the resonances, result in significant deviations in photoionization rates.

In figure 2 we present the percentage difference in the rates, obtained using OP cross sections and the present data, for the three lower states of $\mathrm{O}$ II at radiation temperatures ranging from $10^{3}$ to $10^{5} \mathrm{~K}$. While there are little differences for the ground-state ${ }^{4} \mathrm{~S}^{\mathrm{O}}$, because the cross sections agree very well in the position of the resonances as well as in the resolution between the two sets of data, the differences for the two metastable states are quite significant, up to $30 \%$ for ${ }^{2} \mathrm{P}^{\mathrm{o}}$ and up to $55 \%$ for the ${ }^{2} \mathrm{D}^{\mathrm{o}}$. In order to ascertain whether the differences are due to resolution, or accuracy as reflected in the different positions of resonances, another set of rates is obtained on shifting the resonance positions by a slight amount to match in the two sets of data. This reduces the differences by $10 \%$, with new maximum differences of $20 \%$ and $45 \%$. Resolution also plays a role in the discrepancy. For the metastable states $\left({ }^{2} \mathrm{P}^{\mathrm{o}},{ }^{2} \mathrm{D}^{\mathrm{o}}\right)$, the first resonance is much broader in 
the OP data. The areas under this first peak are 1.8 and 1.9 times higher respectively for the OP cross sections compared to the present set. This affects

the low temperature regime more severely since the first resonance is crucial in convolving with the Planck function, although less so than with a Maxwellian, considered next.

\subsection{Recombination rates}

A similar situation exists for the highly charged ion Li-like O VI. In previous calculations it was shown that the large KLL resonances were severely under-resolved in previous works and a very high resolution is required in order to obtain converged values for the resonance oscillator strengths. The peak values of resonances rise more than four orders of magnitude above the background cross sections. Moreover, since the core ion is He-like, with a large radiative decay rate for the dominant core $1 \mathrm{~s} 2 \mathrm{p}\left({ }^{1} \mathrm{P}^{\mathrm{o}}\right) \rightarrow 1 \mathrm{~s}^{2}\left({ }^{1} \mathrm{~S}_{0}\right)$ transition, radiation damping is important (Zhang et al 1999). This is particularly so in the calculation of unified photo-recombination rate coefficients for (e + O VII) $\leftrightarrow \mathrm{O}$ VI $+h v$ (Nahar and Pradhan 2003b). The unified scheme to obtain total recombination rates, including $\mathrm{RR}$ and DR, entails: (A) highly resolved calculations of photoionization cross sections of a large number of bound levels, typically several hundred levels with effective quantum number $v \leq 10$ and (B) DR calculations using an adaptation of the theory by Bell and Seaton (1985), (Nahar and Pradhan 1994a) for $10<v \leq \infty$. In addition, for any given ion self-consistency is assured in an ab initio manner between total photoionization and recombination since the same wavefunction expansion is employed for both processes (see Nahar (2004), and references therein). Another advantage of the method is that level-specific cross sections and rates for all levels with $v \leq 10$ are also obtained.

Total and level-specific rate coefficients for recombination to O VI and O VII have been reported by Nahar and Pradhan (2003b). In a recent work, Ramirez and Bautista (2002) calculated photoionization cross sections for the ground-state of highly charged ions such as $\mathrm{O}$ VI and $\mathrm{Fe}$ XXIV using a method of locating resonances for high-resolution (Quigley and Berrington 1996), but neglecting effects such as fine structure and radiation damping. Therefore their photo-recombination rate coefficients are in error by large amounts, as seen in figure 3; there is over an order of magnitude discrepancy relative to Nahar and Pradhan (2003b). Thus in this case it is seen that both resolution and accuracy, as reflected in the neglected physical effects, are important. In their work Ramirez and Bautista (2002) discuss the earlier photoionization and (e + ion) recombination calculations for Fe XVII by Pradhan et al (2001); however their uncertainty estimates of ' $25-50 \%$ ' for 'integrated resonance contributions' are not derived from direct computations and are much higher than those computed by Zhang et al (2001).

\subsection{Opacity}

Finally, we investigate these effects on a larger scale, in the total opacity of O II. Figure 4 shows the monochromatic bound-free (bf) opacity due to O II (in atomic units) at a representative stellar

temperature of $2 \times 10^{4} \mathrm{~K}$ and electron density of $10^{17} \mathrm{~cm}^{-3}$, computed as described in Seaton et al (1994)

$$
\kappa^{\mathrm{bf}}(v)=\sum_{i} N_{i} \sigma_{i}^{\mathrm{bf}}(v)\left[1-\mathrm{e}^{-\frac{h v}{k_{\mathrm{B}} T}}\right]
$$

The top panel shows results using the present high-resolution photoionization data for 329 bound 
states of O II, compared with results from OP data. At this temperature and density, 197 bound states of O II contribute to the bf-opacity in the present calculations and 169 in the OP data. The difference in the number of bound states is inconsequential; the additional states in the present work are mostly highly excited ones. The monochromatic opacity is sampled

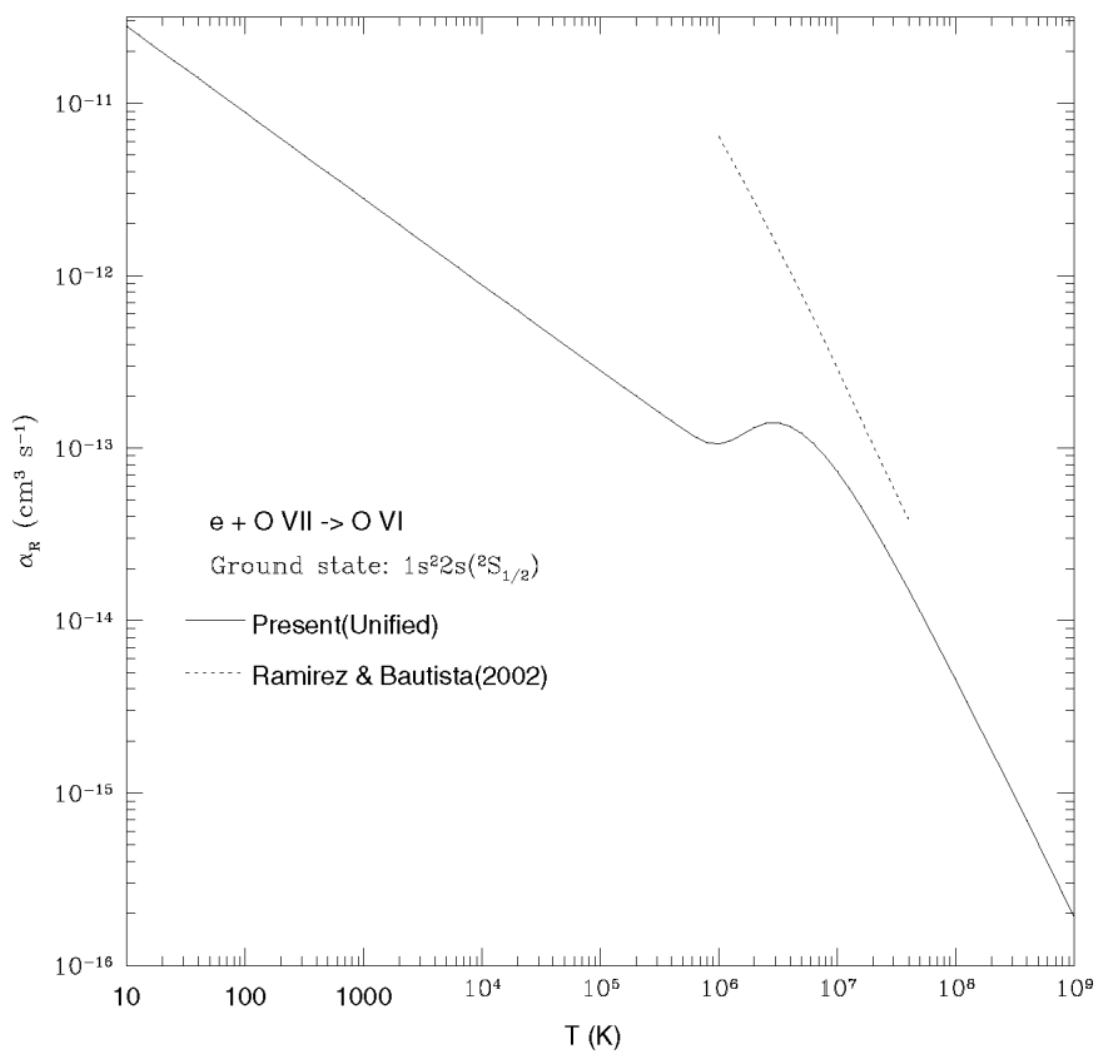

Figure 3. Photo-recombination rate coefficient for the ground-state of O VI $\left(1 \mathrm{~s}^{2} 2 \mathrm{~s}^{2} \mathrm{~S}_{1 / 2}\right)$ : solid line - using cross sections from Nahar and Pradhan (2003), dashed line-Ramirez and Bautista (2002); the differences are due to the neglect of radiation damping in the latter calculations.

at $10^{5}$ points in both calculations. As figure 4 shows, there is overall very good agreement between the two sets, despite the significantly better resolution and accuracy of the present calculations. This is expected since strong features dominate the opacity spectrum and are found in both sets of calculations. The present Rosseland and Planck mean bf-opacities

$$
\begin{aligned}
\frac{1}{\kappa_{R}^{\mathrm{bf}}} & =\int \frac{1}{\kappa_{v}^{\mathrm{bf}}} F_{R}(v) \mathrm{d} v \\
\kappa_{P}^{\mathrm{bf}} & =\int \kappa_{v}^{\mathrm{bf}} F_{P}(v) \mathrm{d} v
\end{aligned}
$$

where 


$$
\begin{aligned}
& F_{R}(v)=\frac{10}{4 \pi^{4}}\left(\frac{n v}{k_{\mathrm{B}} T}\right) \frac{\mathrm{e}^{{ }^{k_{\mathrm{B}}} 1}}{\left(1-\mathrm{e}^{-\frac{h v}{k_{\mathrm{B}} T}}\right)^{2}} \\
& F_{P}(v)=\frac{15}{4 \pi^{5}}\left(\frac{h v}{k_{\mathrm{B}} T}\right)^{3} \frac{\mathrm{e}^{-\frac{h v}{k_{\mathrm{B}} T}}}{\left(1-\mathrm{e}^{-\frac{h v}{k_{\mathrm{B}} T}}\right)}
\end{aligned}
$$

are 0.159 and 2.12 respectively, compared to 0.146 and 2.42 from the OP data, i.e. the Rosseland means are $8 \%$ higher and the Planck means are $14 \%$ lower than OP (electron scattering opacity

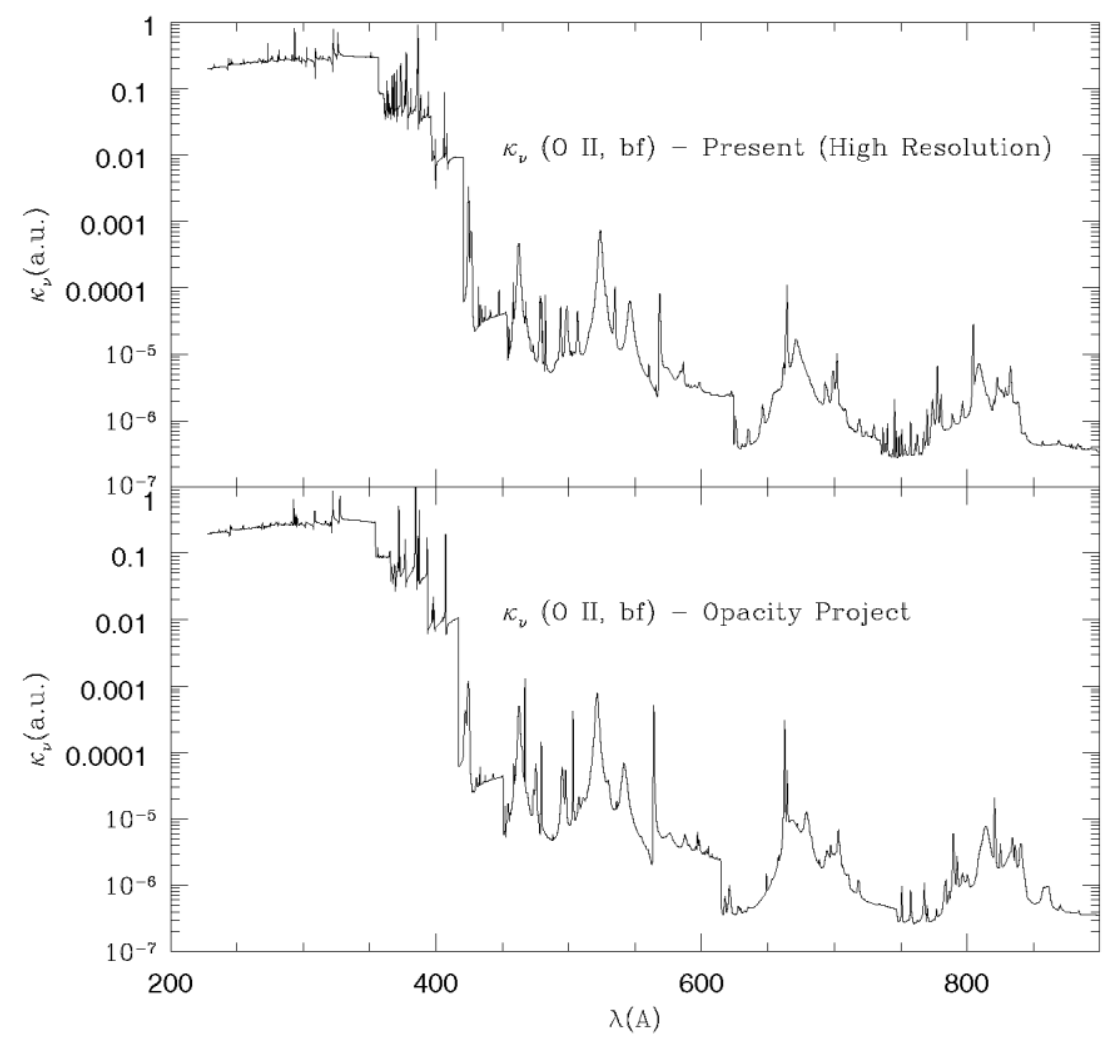

Figure 4. Monochromatic bound-free opacity of O II using the present high-resolution atomic data for photoionization cross sections of 329 bound states (upper panel) and using the Opacity Project data for the same states (lower panel).

is included to provide a background and is the same in both calculations). In the present opacity calculations the resonances are better resolved and in many cases narrower compared to the OP data, which results in a smaller Planck mean; conversely, since the minima in resonances are better sampled, the Rosseland mean is slightly higher. However, the total Rosseland mean opacities differ only by $2.6 \%$, if we include the same (much larger) bound-bound contribution from lines in the two calculations -0.345 from the present versus 0.336 from the OP data. 


\section{Conclusion}

In conclusion we note that

1. For photoionization and NLTE modelling applications, it may be necessary to compute photoionization cross sections with higher accuracy and resolution than in the Opacity Project data. Accuracy of resonance positions is crucial in the determination of $(e+$ ion) and photoionization rates. A number of self-consistent computations are in progress as part of a programme to calculate photoionization and unified $(\mathrm{e}+$ ion) recombination (RR plus DR) cross sections and rates (Nahar and Pradhan 2003a).

2. Although the monochromatic and mean opacities are not measurably different using the high-resolution data for oxygen ions, as opposed to the Opacity Project data, these

quantities may differ considerably for more complex atomic systems such as the low ionization stages of iron. In a previous work (Nahar and Pradhan 1994b), we showed that the Rosseland mean opacities using new improved data for Fe II differed by up to 50\% from OP values. There is increasing evidence that there is 'missing UV opacity', possibly due to Fe I, that is critical to the determination of abundances in the Sun and other objects (e.g. Asplund 2003). New atomic and opacity calculations for iron ions are needed with higher accuracy and resolution.

\section{Acknowledgments}

This work was partially supported by the US National Science Foundation and NASA. The computational work was carried out on the Cray SV1 at the Ohio Supercomputer Center in Columbus, Ohio.

\section{References}

Asplund M 2003 Preprint astro-ph 0312291 (Astron. Astrophys. at press)

Bell R H and Seaton M J 1985 J. Phys. B: At. Mol. Phys. 181589

Berrington K A, Eissner W and Norrington P H 1995 Comput. Phys. Commun. 92290

Burke P G, Hibbert A and Robb W D 1971 J. Phys. B: At. Mol. Phys. 4153

Burke P G and Berrington K A 1993 Atomic and Molecular Processes: an R-matrix Approach (Bristol: Institute of Physics Publishing)

Champeaux J-P, Bizau J-M, Cubaynes D, Blancard C, Nahar S N, Hitz D, Bruncau J and Wuilleumier F J 2003 Astrophys. J. Supp. Ser. 148583

Covington A M et al 2001 Phys. Rev. Lett. 87243002

Cunto W, Mendoza C, Ochsenbein F and Zeippen C J 1993 Astron. Astrophys. 275 L5

Hummer D G, Berrington K A, Eissner W, Pradhan A K, Saraph H E and Tully J A 1993 Astron. Astrophys. 279298

Kjeldsen H, Folkmann F, Hensen J E, Knudsen H, Rasmussen M S, West J B and Andersen T 1999 Astrophys. J. 524 L143

Nahar S N 2002 Phys. Rev. A 655207

Nahar S N 2004 Phys. Rev. A 69042714

Nahar S N and Pradhan A K 1992 Phys. Rev. Lett. 681488

Nahar S N and Pradhan A K 1994a Phys. Rev. A 491816

Nahar S N and Pradhan A K 1994b J. Phys. B: At. Mol. Opt. Phys. 27429

Nahar S N and Pradhan A K 2003a Self-consistent $R$-matrix approach to photoionization and unified electron-ion recombination Radiation Processes in Physics and Chemistry (Amsterdam: Elsevier) at press

Nahar S N and Pradhan A K 2003b Astrophys. J. Suppl. Ser. 149239

Pradhan A K 2000 Astrophys. J. 545 L165

Pradhan A K, Nahar S N and Zhang H L 2001 Astrophys. J. 549 L265

Quigley L and Berrington K A 1996 J. Phys. B: At. Mol. Opt. Phys. 294529

Ramirez J M and Bautista M A 2002 J. Phys. B: At. Mol. Opt. Phys. 354139 
Seaton M J, Yu Y, Mihalas D and Pradhan A K 1994 Mon. Not. R. Astron. Soc. 266805

The Opacity Project Team 1995/1996 The Opacity Project, vols 1 and 2 (Bristol: Institute of Physics Publishing) Zhang H L and Pradhan A K 1997 Phys. Rev. Lett. 78195

Zhang H L, Nahar S N and Pradhan A K 1999 J. Phys. B: At. Mol. Opt. Phys. 321459

Zhang H L, Nahar S N and Pradhan A K 2001 Phys. Rev. A 64032719 